\title{
Genetic screening of Congenital Short Bowel Syndrome patients confirms CLMP as the major gene involved in the recessive form of this disorder
}

\author{
Maria M Alves ${ }^{\star 1}$, Danny Halim ${ }^{1,10}$, Reza Maroofian ${ }^{2,10}$, Bianca M de Graaf ${ }^{1}$, Raoul Rooman ${ }^{3,11}$, \\ Christine S van der Werf ${ }^{4}$, Els Van de Vijver ${ }^{3}$, Mohammad YV Mehrjardi ${ }^{5,6}$, Majid Aflatoonian ${ }^{7}$, \\ Barry A Chioza ${ }^{2}$, Emma L Baple ${ }^{2,8}$, Mohammadreza Dehghani ${ }^{5,9}$, Andrew H Crosby ${ }^{2}$ and Robert MW Hofstra ${ }^{1}$
}

Congenital short bowel syndrome (CSBS) is an intestinal pediatric disorder, where patients are born with a dramatic shortened small intestine. Pathogenic variants in CLMP were recently identified to cause an autosomal recessive form of the disease. However, due to the rare nature of CSBS, only a small number of patients have been reported to date with variants in this gene. In this report, we describe novel inherited variants in CLMP in three CSBS patients derived from two unrelated families, confirming CLMP as the major gene involved in the development of the recessive form of CSBS.

European Journal of Human Genetics (2016) 24, 1627-1629; doi:10.1038/ejhg.2016.58; published online 29 June 2016

\section{INTRODUCTION}

During embryogenesis, the small intestine experiences major growth expanding several times the total length of the whole body. This is a complex process that starts around the fifth week of human development, when the midgut forms a simple linear tube that runs down the midline of the embryo, and is completed by the twentieth week of development. ${ }^{1}$ Several pathways have been reported to have a role in intestinal elongation, ${ }^{2}$ but to date the molecular mechanism(s) responsible for this process remains unclear.

In congenital short bowel syndrome (CSBS), intestinal elongation is impaired and patients are born with a substantially shortened small intestine ( $\sim 50 \mathrm{~cm}$ in length instead of $250 \mathrm{~cm}$ in a normal counterpart). ${ }^{3}$ It is a rare disorder for which no cure is available. Total parenteral nutrition is required for long-term survival, but despite considerable efforts to improve treatment, most patients die of starvation or sepsis within the first few days of life. ${ }^{4}$ CSBS was first described by Hamilton et al. ${ }^{3}$ and it has always been considered to have a genetic cause. $^{3}$ However, it was only recently that two genes were identified as the cause of CSBS: CLMP and FLNA. ${ }^{5,6}$ CLMP encodes for the Coxsackie and adenovirus receptor-like membrane protein, an adhesion molecule that co-localizes with tight junction proteins, but whose function is still unknown. ${ }^{7}$ FLNA encodes for Filamin-A, an actinbinding protein known to regulate cell shape and to control cell signaling and migration. ${ }^{8}$ Pathogenic variants present in CLMP and FLNA have been linked to the recessive and X-linked forms of CSBS, respectively.

Here, we report the genetic screening of three CSBS patients derived from two unrelated families, and describe the identification of three novel variants in $C L M P$.

\section{MATERIAL AND METHODS}

Patient information

In this study, three female patients diagnosed with CSBS were investigated (Table 1). Two of these patients were siblings of consanguineous descent (P1 and P2). The third patient (P3) has been previously described, when a de novo translocation was found between chromosome 2 and $11(46, \mathrm{XX}, \mathrm{t}(2,11)(\mathrm{q} 32.2, \mathrm{p} 12)) .{ }^{9}$ However, no gene was identified as the causative factor at that time. A complete description of the patients can be found in Supplementary Data.

Written informed consent was given by the two families reported, and ethical approval was obtained from the Erasmus Medical Center ethical committee (Medisch Ethische Toetsings commissie - METc 2009/364, ABR nr: NL31708.042.10).

\section{Genetic analysis}

Genomic DNA was isolated from peripheral blood lymphocytes using standard methods. Exons 1-7 of CLMP (ENST00000448775) were amplified using $30 \mathrm{ng}$ of genomic DNA as described before. ${ }^{5}$ PCR products were purified (ExoSap-IT - GE Healthcare, Eindhoven, The Netherlands), and Sanger sequencing was performed with dye labeled primers (forward and reverse; Big Dye Terminator v3.1 Sequencing Kit, Applied Biosystems, Bleiswijk, The Netherlands) on an ABI 3130XL genetic analyzer (Applied Biosystems). Sanger reads were analyzed using SeqScape software (Fisher Scientific, Landsmeer, The Netherlands) and compared with the reference CLMP genomic sequence (ENSG00000166250). Genetic data were submitted to ClinVAr (http://www.ncbi.nlm.nih.gov/clinvar/) and the following

\footnotetext{
${ }^{1}$ Department of Clinical Genetics, Erasmus University Medical Center, Rotterdam, The Netherlands; ${ }^{2}$ University of Exeter Medical School, RILD Wellcome Wofston Centre, Royal Devon \& Exeter NHS Foundation Trust, Exeter, UK; ${ }^{3}$ Department of Pediatrics, University Hospital of Antwerp, Antwerp, Belgium; ${ }^{4}$ Department of Genetics, University Medical Center Groningen, Groningen, The Netherlands; ${ }^{5}$ Biomedical and Clinical Genetic Research Center, Shahid Sadoughi University of Medical Sciences, Yazd, Iran; ${ }^{6}$ Department of Medical Genetics, Shahid Sadoughi University of Medical Sciences, Yazd, Iran; ${ }^{7}$ Department of Pediatrics, Shahid Sadoughi University of Medical Sciences, Yazd, Iran; ${ }^{8}$ Faculty of Medicine, University of Southampton, Southampton, UK; ${ }^{9}$ Research and Clinical Center for Infertility, Shahid Sadoughi University of Medical Sciences, Yazd, Iran

*Correspondence: Dr MM Alves, Department of Clinical Genetics, Erasmus University Medical Center, PO Box 2040, 3000 CA Rotterdam, The Netherlands. Tel +31-10-7037643; E-mail: m.alves@erasmusmc.nl

${ }^{10}$ These authors contributed equally to this work.

${ }^{11}$ Current address: PendoCon, Putte, Belgium.

Received 16 October 2015; revised 23 April 2016; accepted 4 May 2016; published online 29 June 2016
} 
Table 1 Clinical and molecular features of the CSBS patients included in this study

\begin{tabular}{|c|c|c|c|}
\hline & P1 & P2 & P3 \\
\hline Gender & Female & Female & Female \\
\hline Age of diagnostic & 3 months & 38 days & 1 week \\
\hline Ethnicity & Iranian & Iranian & Caucasian \\
\hline Consanguinity & + & + & No \\
\hline Malrotation & + & + & + \\
\hline Length of the small intestine $(\mathrm{cm})$ & 76 & 26 & 50 \\
\hline Additional features & Intestinal dysmotility UPJO & Intestinal dysmotility UPJO & Very mild mental retardation \\
\hline CLMP variants & c. $508 \mathrm{C}>\mathrm{T} ; \mathrm{p} .\left(\mathrm{R} 170^{*}\right)$ & c. $508 \mathrm{C}>\mathrm{T} ;($ p.R170*) & c. $410 G>A ; p .(C 137 Y)$ c. $29-2 A>G$ \\
\hline Zygosity & Homozygous & Homozygous & Heterozygous (two unphased variants) \\
\hline
\end{tabular}

Abbreviations: CSBS, congenital short bowel syndrome; UPJO, ureteropelvic junction obstruction.

a

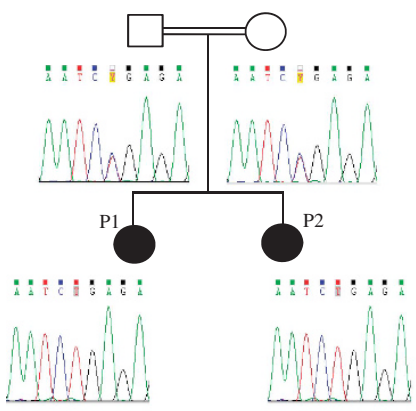

C

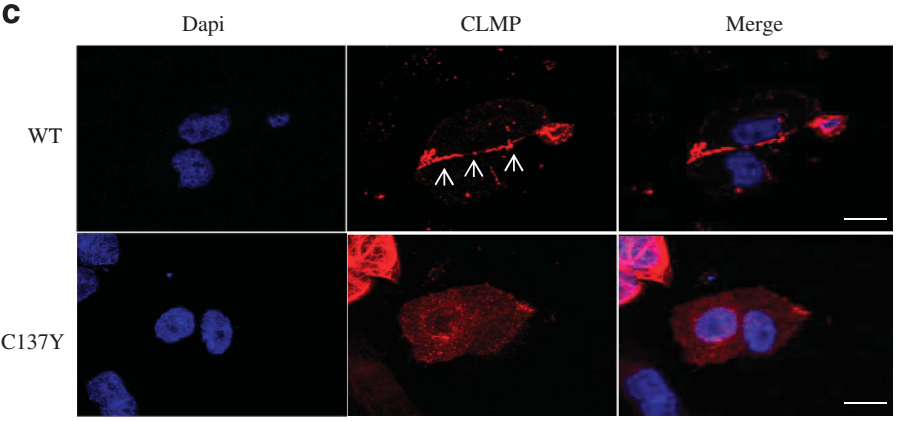

e

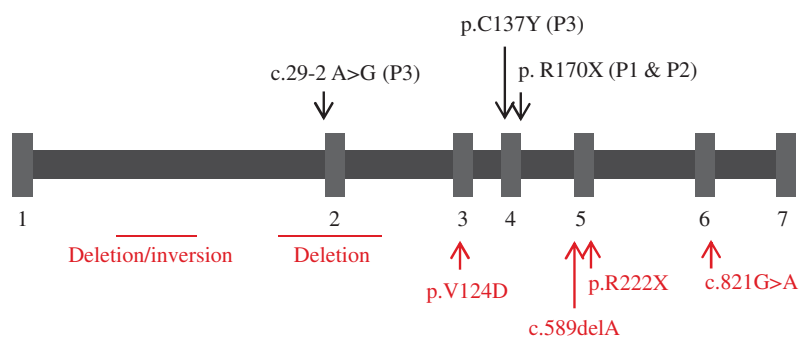

Family 2

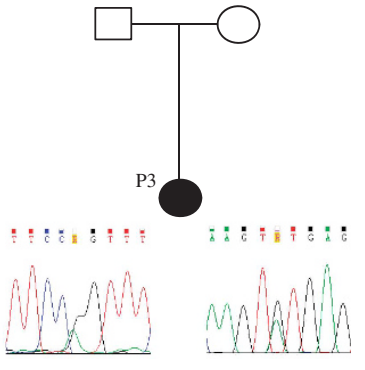

b

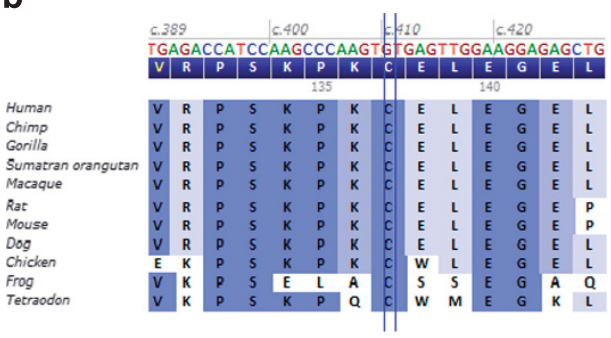

d

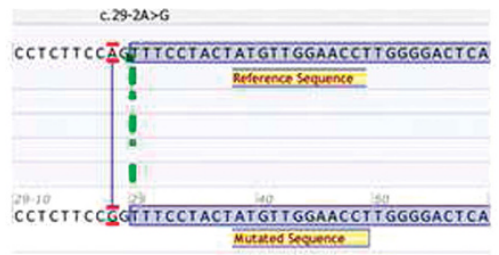

Figure 1 Genetic analysis of three CSBS patients. (a) Pedigree of the two families included in this study with Sanger sequencing results showing the presence of variants in CLMP. (b) Conservation alignments showed that the missense variant $p .(C 137 Y)$ leads to a change of a cysteine residue highly conserved among vertebrates. (c) CHO-K1 cells transfected with constructs expressing wild-type (WT) CLMP or the p.(C137Y) variant show different cellular distribution of CLMP. While the WT protein normally localizes to the tight junctions (see arrows), the mutated protein has a punctuated distribution through the cytoplasm. Scale bars: $30 \mu \mathrm{m}$. (d) Splicing predictions suggested that the intronic variant identified in patient 3 likely affects normal splicing, as it is predicted to disrupt an acceptor-splicing site (c.29-2A $>$ G). (e) Schematic overview of all the variants identified to date in CLMP. In black are the variants reported in this report, whereas in red are the variants previously described. b and $\mathbf{d}$ were generated using Alamut prediction software (Interactive Biosoftware, Rouen, France). 
accession numbers were obtained: SCV000264793 [p.(R170*)] and SCV000264792 [p.(C137Y) and (c.29-2A>G)].

\section{Expression vectors}

Generation of the expression vectors used is described in Supplementary Data.

\section{Immunofluorescence}

Chinese hamster cells (CHO-K1) were cultured as described before. ${ }^{5}$ Five hundred thousand cells were seeded in a 6-well plate, and transiently transfected with pcDNA-HA-CLMP WT or pcDNA-HACLMP C137Y expressing vectors, using GeneJuice (Millipore, Amsterdam, The Netherlands) as transfection reagent. Twenty four hours after transfection, immunofluorescence was performed as previously described. ${ }^{5}$ Images were taken in a Leica (AOBS) microscope, and analyzed with the Leica LAS AF Lite software (Leica Microsystems B. V., Eindhoven, The Netherlands).

\section{RESULTS AND DISCUSSION}

As the three patients included in this study are females, an X-linked pattern of inheritance involving FLNA was considered unlikely, and only CLMP was screened. In all patients, we identified previously unreported variants in CLMP. None of these variants are listed in any of the available human genome variant databases. Patients P1 and P2 possess a homozygous nonsense variant in exon 4 [c.508C $>\mathrm{T}$; $\left.\mathrm{p} .\left(\mathrm{R} 170^{\star}\right)\right]$, leading to the appearance of an early stop codon (Figure 1a). This nonsense variant was inherited from the parents, who were found to be heterozygous. Patient P3 has two unphased heterozygous variants in CLMP. Although we suspect that both variants were inherited from the parents, we were unable to investigate segregation in the family due to unavailability of parental DNA. In this patient, a missense variant located in exon 4 was identified leading to an amino acid substitution [c.410G > A; p. (C137Y)], together with a possible splice site variant located two base pairs upstream of exon two (c.29-2A > G) (Figure 1a). Prediction programs used to infer pathogenicity (PolyPhen-2, MutationTaster, SIFT, Human Splicing Finder and Alamut Visual) showed that both variants are likely to disturb protein function. The C137Y variant is located in a highly conserved region present in all mammals (Figure $1 \mathrm{~b}$ ), and in vitro assays showed that it affects the cellular localization of CLMP to the tight junctions (Figure 1c). The c.29-2A $>\mathrm{G}$ variant is predicted to disrupt normal splicing of exon 2 of CLMP (Figure 1d). Considering that both variants likely affect CLMP function, we believe that the de novo translocation previously identified in patient $\mathrm{P}^{9}$ is an independent event unrelated to CSBS.

Currently, only seven patients have been described carrying pathogenic variants in CLMP. ${ }^{5}$ All the variants reported were loss of function, leading to the absence of CLMP or to the expression of a mutant protein with a disrupted function (Figure 1e). In this report, we expand the mutational spectrum of CLMP variants involved in CSBS, consolidating the involvement of this gene in the development of the recessive form of this disease. We also show that it might be worthwhile to screen previously described patients for whom an $\mathrm{X}$-linked pattern of inheritance was disregarded, for the presence of variants in CLMP, as this might shed some light on the genetic basis of the disease in these cases. To date, the role of CLMP in intestinal elongation is still largely unknown, making it difficult to determine the molecular mechanisms underlying the development of CSBS. We believe that the results here described reinforce the need to further study the role of CLMP in intestinal elongation, as this might contribute for the development of new therapeutic strategies for CSBS patients.

\section{CONFLICT OF INTEREST}

The authors declare no conflict of interest.

\section{ACKNOWLEDGEMENTS}

We would like to thank all the families involved in this study. We would also like to thank Katherine MacKenzie for technical assistance with the confocal microscope. In addition, we would like to dedicate this short report in memory of the pediatric gastroenterologist involved in the diagnosis and care of one of the patients described, Dr André Deprettere. This study was supported by a grant from the Stichting Sophia Kinderziekenhuis Fonds (MMA and RMWH), a Mrace grant from the Erasmus University Medical Center (RMWH), and by the Newlife Foundation, as well as the MRC, UK (AHC).

1 Moore KL, Persaud TVN: The Developing Human: Clinically Oriented Embryology. Philadelphia: Saunders, 2003.

2 van der Werf CS, Halim D, Verheij JB, Alves MM, Hofstra RM: Congenital short bowel syndrome: from clinical and genetic diagnosis to the molecular mechanisms involved in intestinal elongation. Biochim Biophys Acta 2015; 1852: 2352-2361.

3 Hamilton JR, Reilly BJ, Morecki R: Short small intestine associated with malrotation: a newly described congenital cause of intestinal malabsorption. Gastroenterology 1969; 56: 124-136.

4 Howard L, Ament M, Fleming CR, Shike M, Steiger E: Current use and clinical outcome of home parenteral and enteral nutrition therapies in the United States. Gastroenterology 1995; 109: 355-365.

5 van der Werf CS, Wabbersen TD, Hsiao NH et al: CLMP is required for intestinal development, and loss-of-function mutations cause congenital short-bowel syndrome. Gastroenterology 2012; 142: 453-462.

6 van der Werf CS, Sribudiani Y, Verheij JB et al: Congenital short bowel syndrome as the presenting symptom in male patients with FLNA mutations. Genet Med 2013; 15: 310-313.

7 Raschperger E, Engstrom U, Pettersson RF, Fuxe J: CLMP, a novel member of the CTX family and a new component of epithelial tight junctions. J Biol Chem 2004; 279 : 796-804.

8 Robertson SP, Filamin A: Phenotypic diversity. Curr Opin Genet Dev 2005; 15 : 301-307.

9 de Backer Al, Parizel PM, de Schepper A, Vaneerdeweg W: A patient with congenita short small bowel associated with malrotation. J Belge Radiol 1997; 80: 71-72.

Supplementary Information accompanies this paper on European Journal of Human Genetics website (http://www.nature.com/ejhg) 